\title{
Identification of a Novel Protein Kinase A Inhibitor by Bioluminescence- Based Screening
}

\author{
Tetsuya Ishimoto,* Kenji Azechi, and Hisashi Mori \\ Department of Molecular Neuroscience, Graduate School of Medicine and Pharmaceutical Sciences, University of \\ Toyama; 2630 Sugitani, Toyama 930-0194, Japan. \\ Received July 16, 2015; accepted September 15, 2015
}

\begin{abstract}
We screened inhibitors in the adenylyl cyclase/protein kinase A/cAMP response element binding protein pathway (AC/PKA/CREB pathway) from a 2400 chemical library by a cell-based assay method using bioluminescence probes. We found a compound that inhibited forskolin-induced cAMP response element (CRE)dependent transcription, the interaction between the kinase-inducible domain (KID) and the interacting domain (KIX), and endogenous CREB phosphorylation. Furthermore, this compound suppressed the activity of the PKA catalytic subunit dose-dependently. On the other hand, this compound did not inhibit forskolininduced CAMP up-regulation. Taken together, we conclude that we have identified a new PKA inhibitor that binds to the catalytic subunit directly. We also succeeded in shortening the screening protocol by excluding a screening step which was used in a previous method.
\end{abstract}

Key words protein kinase A; high-throughput screening; inhibitor; split luciferase

Activation of the adenylyl cyclase/protein kinase A/cAMP response element binding protein $(\mathrm{AC} / \mathrm{PKA} / \mathrm{CREB})$ pathway is considered to be necessary for the expression of the proteins required for memory consolidation. ${ }^{1,2)}$ However, overactivation of CREB is seen in the brain of patients with posttraumatic stress disorder ${ }^{3)}$ and addiction. ${ }^{4)}$ Moreover elevated activation of CREB has been demonstrated in some tumor cells. ${ }^{5-7)}$ Thus it is considered that inhibitors of this pathway may be used as medicines for these diseases.

Previously, we screened inhibitors of the proteins in the AC/PKA/CREB pathway using cultured cells expressing split luciferase probe proteins from the 2400 chemical library and identified an $\mathrm{AC}$ inhibitor. ${ }^{8)}$ The split luciferase technique is used for detecting protein-protein interaction using firefly luciferase. ${ }^{9)}$ In this technique, cleaved segments of firefly luciferase (lucN and lucC) are fused with the kinase inducible domain (KID) of CREB and the interacting domain (KIX) of the CREB-binding protein (CBP). Following the phosphorylation of KID, which results in CREB activation, KID interacts with KIX, and split segments (lucN and lucC) complement each other to be a functional luciferase that can emit light. ${ }^{10)}$ Thus, when the cells expressing these probe proteins receive a stimulus that activates the AC/PKA/CREB pathway such as forskolin, they show an increased light intensity. However, the inhibitors of proteins in $\mathrm{AC} / \mathrm{PKA} / \mathrm{CREB}$ pathway cancel this increase.

So far, many inhibitors of the AC/PKA/CREB pathway have been identified. However finding new inhibitors is still demanded because most of the known inhibitors have side effects such as inhibition of kinases in other pathways ${ }^{11)}$ and are not suitable for medical use. In this study, we attempt to further explore inhibitors of the AC/PKA/CREB pathway using another set of a chemical library consisting of 2400 compounds in addition to the library previously used. ${ }^{8)} \mathrm{We}$ also tried to shorten the screening method by omitting a screening step to exclude inhibitors for wild type luciferase which was used in previous paper, since few compound inhibited wild type luciferase. ${ }^{8)}$

\section{MATERIALS AND METHODS}

Cell Culture and Transfection For the first screening, HEK293 cell stably expressing KID-KIX probes were used. Cells were grown in Dulbecco's modified Eagle's medium (DMEM) containing 10\% fetal calf serum in 96-well plates at $37^{\circ} \mathrm{C}$ in an incubator with $5 \% \mathrm{CO}_{2}$. HEK293T cells were used in other experiments under the same culture condition as HEK293 cells.

KID-KIX Probe Assay The culture medium of HEK293T expressing probe proteins were exchanged with L15 containing $0.5 \mathrm{~mm}$ luciferin (AAT Bioquest, Sunnyvale, CA, U.S.A.) and incubated for $1 \mathrm{~h}$ at room temperature. Then, cells were treated with each of the 2400 compounds $(10 \mu \mathrm{M})$ provided by the University of Tokyo (Core Library, Open Innovation Center for Drug Discovery) for $2 \mathrm{~h}$, and then with forskolin $(10 \mu \mathrm{M}$, Sigma-Aldrich, St. Louis, MO, U.S.A.) for $4 \mathrm{~h}$. The intensity of light emitted from cells was measured using an Aequoria-2D/C8600 system (Hamamatsu Photonics, Hamamatsu, Japan) and Wasabi software (Hamamatsu Photonics). The compounds that suppressed the increase in light emission intensity induced by forskolin were subjected to the next assay.

CRE-Luc Assay HEK293T cells were transfected with pGL4.29 (Promega, Madison, WI, U.S.A.) 1d after passage according to the manual for the use of lipofectamine 2000 (Invitrogen, Carlsbad, CA, U.S.A.). Two days after transfection, the medium was exchanged with L15 containing $0.5 \mathrm{~mm}$ luciferin and incubated at room temperature for $1 \mathrm{~h}$. Then, the cells were treated with each of the compounds $(10 \mu \mathrm{M})$ for $2 \mathrm{~h}$ and then with forskolin $(10 \mu \mathrm{M})$ for $12 \mathrm{~h}$. Relative changes in light emission intensity from each well were calculated.

Western Blotting Cells were lysed with M-PER solution (Thermo Scientific, Waltham, MA, U.S.A.). Extracted proteins were separated by sodium dodecyl sulfate-polyacrylamide gel electrophoresis (SDS-PAGE) and electrically transferred onto a polyvinylidene difluoride (PVDF) membrane (GE Healthcare, Pittsburg, PA, U.S.A.). The membrane was incubated 
with a rabbit anti-pCREB $(1: 500$, Cell Signaling Technology, Boston, MA, U.S.A.) or rabbit anti-CREB (1:500, Cell Signaling Technology) primary antibody in the Can Get Signal solution (Toyobo, Tokyo, Japan) at $4{ }^{\circ} \mathrm{C}$ overnight. The membrane was reacted with a secondary goat anti-rabbit immunoglobulin $\mathrm{G}$ (IgG) horseradish peroxidase (HRP)-conjugated antibody (1:5000, Bio Rad, Hercules, CA, U.S.A.) and visualized using an ECL Prime Western blotting reagent (GE Healthcare). Visualization and quantification were performed using a LAS4000 system (GE Healthcare).

PKA Assay A pep-tag PKA assay kit (Promega) was used to measure the inhibitory effect of the candidate compound on PKA. The bovine PKA catalytic subunit $(0.4 \mu \mathrm{g} / \mathrm{mL})$ supplied in the kit was incubated with the compound at several doses for $15 \mathrm{~min}$ at room temperature. Then the fluorescence-tagged PKA substrate was added to the solution and incubated in the buffer containing $20 \mathrm{~mm}$ Tris $\mathrm{pH} 7.4,10 \mathrm{~mm} \mathrm{MgCl}_{2}$, and $1 \mathrm{~mm}$ or $0.2 \mathrm{~mm}$ ATP for $30 \mathrm{~min}$ at $37^{\circ} \mathrm{C}$, and PKA was heatinactivated $\left(95^{\circ} \mathrm{C}, 10 \mathrm{~min}\right)$. The PKA substrate peptide was electrophoresed in $0.8 \%$ agarose gel for $15 \mathrm{~min}$ at $100 \mathrm{~V}$. During the electrophoresis, a phosphorylated peptide moved to the cathode, whereas nonphosphorylated peptide moved to the anode. Bands corresponding to substrate peptides were quantified under ultraviolet light illumination using a LAS4000 system (GE Healthcare). The percentage of the phosphorylated substrate was calculated from the signal intensity of phosphorylated substrate with respect to that of total substrate. The $\mathrm{IC}_{50}$ value of the candidate compound in the assays was calculated by fitting of the Hill equation to the dose-response data.

cAMP Assay The concentration of cAMP was measured using a DetectX Direct Cyclic AMP Enzyme Immunoassay kit (ARBOR ASSAYS, Ann Arbor, MI, U.S.A.) in accordance with manufacturer's instruction. HEK293T cells were treated with the candidate compound $(200 \mu \mathrm{M})$ for $1 \mathrm{~h}$, and then with forskolin $(10 \mu \mathrm{M})$ for $15 \mathrm{~min}$. The cells were rinsed with phosphate buffered saline (PBS) and harvested using sample diluent supplied in the kit. After the centrifugation $(600 \times \mathbf{g}$, $15 \mathrm{~min}$ ), the resulting supernatant was used for the reaction. After the reaction, optical density at $450 \mathrm{~nm}$ was measured and fitted to the standard curve to calculate cAMP concentration.

Cell Viability Assay Cell viability was determined by 3-(4,5-dimethyl-2-thizolyl)-2,5-diphenyl-2H-tetrazolium bromide (MTT) assay. HEK293T cells were incubated with MTT $(1 \mathrm{mg} / \mathrm{mL})$ for $1 \mathrm{~h}$ at room temperature, and the deposited MTT were solubilized with $1 \mathrm{~mL}$ of dimethyl sulfoxide. Absorbance at wavelengths of 570 and $630 \mathrm{~nm}$ was measured using a spectrophotometer (Gene Quant 1300, GE Healthcare). Sample signal intensity was obtained by subtracting optical density (OD) at $630 \mathrm{~nm}$ from that at $570 \mathrm{~nm}$ and indicated as a percentage of the control value.

Quantitative Polymerase Chain Reaction (PCR) Analysis Total RNA purified from HEK293T cells using an RNeasy mini kit (Qiagen Hilden, Germany) was reverse-transcribed using ReverTra Ace (Toyobo). The MX3005P system (Agilent Technologies, Palo Alto, CA, U.S.A.) with the Thunderbird SYBR qPCR Mix (Toyobo) was used in the comparative quantification mode. The $\mathrm{Ct}$ of glyceraldehyde-3-phosphate dehydrogenase (GAPDH) was used for the calculation of the relative quantity of $c$-fos mRNA. The primers used were $5^{\prime}$-gtcggagtcaacggatttgg-3' and 5'-catggaatttgccatgggtg-3' for
Gapdh, and 5'-ctgtggettccettgatctg-3' and $5^{\prime}$-tgetcttgacaggttcca ct-3' for $c$-fos.

\section{RESULTS AND DISCUSSION}

Previously, we screened 2400 compounds and found an AC inhibitor using a cell-based screening method. ${ }^{8)}$ In the current study, we started a new series of screening with another 2400 compounds using the same screening method. HEK293 cells stably expressing KID-KIX probe proteins were stimulated with forskolin $(10 \mu \mathrm{M})$ in the presence of one of the compounds $(10 \mu \mathrm{M})$ of the chemical library. Since KID-KIX probe proteins mimic phosphorylation-dependent CREB-CBP interaction, an increase in light emission intensity means activation of the AC/PKA/CREB pathway. We found 51 compounds that suppressed the increase in light intensity in response to the forskolin treatment from cells expressing KID-KIX probe proteins. From this finding, it is considered that the 51 compounds blocked the activity of proteins in the AC/PKA/ CREB pathway. We performed CMV-luc assay to test whether the compounds inhibited wild type luciferase activity after KID-KIX probe assay in the previous report, however, CMVluc assay was excluded in current screening to simplify the screening. Next, these 51 compounds were subjected to the second screening using HEK293T cells carrying the plasmid that contained the CRE sequence in the promotor region of luciferase. HEK293T cells expressing luciferase depending on CRE (CRE-luc) were treated with the 51 compounds, subsequently with forskolin. Most of the cells showed the increase in light emission intensity in response to forskolin, whereas cells treated with a compound (candidate compound) did not (Fig. 1A). The structure of the candidate compound is shown in Fig. 1B. From these result, the candidate compound (2-naphthalenesulfonamide, $\quad N$-methyl- $N$-[(1,3,5-trimethyl- $1 H$ -

A)

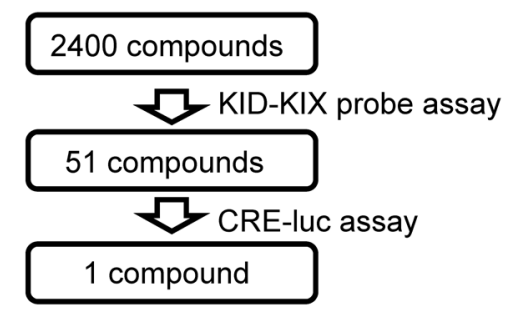

B)

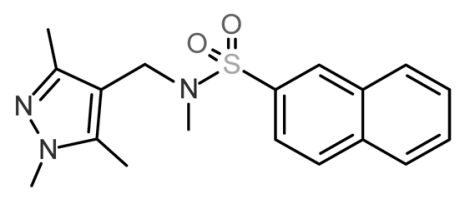

Fig. 1. Schematic Diagram of Screening Process

A) Screening was started using 2400 compounds in the first step. KID-KIX probe assay and CRE-luc assay were carried out to narrow down the compounds. B) Chemical structure of identified candidate compound. 
pyrazol-4-yl)methyl])-inhibited CRE-dependent gene expression. And also, we succeeded in shortening the screening method by excluding the CMV-luc step used in previous paper.

To validate the results of first and second screenings, we examined the effect of the candidate compound on the light emission from the KID-KIX probe proteins and CRE-luc again. We found that the candidate compound completely inhibited the forskolin-induced increase in the intensity of light emission from HEK293T cells expressing the KID-KIX probe protein (Fig. 2A) and CRE-luc plasmid (Fig. 2B). From this validation, we concluded that the candidate compound reproducibly inhibited proteins in the $\mathrm{AC} / \mathrm{PKA} / \mathrm{CREB}$ pathway. Since forskolin-induced activation of the AC/PKA/CREB pathway consists of several steps, as shown in Fig. $2 \mathrm{C}$, the candidate compound may inhibit not only KID-KIX binding but also upstream proteins involved in the pathway. Next, we attempted to elucidate the target of the candidate compound.

We tested whether the candidate compound inhibits the phosphorylation of endogenous CREB in response to forskolin $(10 \mu \mathrm{M})$ treatment. HEK293T cell treated with or without the candidate compound were stimulated with forskolin and harvested and analyzed by Western blotting using anti-pCREB and anti-total CREB antibodies. Forskolin-induced CREB phosphorylation was apparently inhibited in the candidatecompound $(10 \mu \mathrm{M})$-treated cells, whereas cells treated with a previously reported KID-KIX binding inhibitor ${ }^{12)}$ was not (Figs. 3A, B). This result suggests that the candidate compound inhibits the activity of PKA or its upstream enzyme AC.

Then, the inhibitory effect of the candidate compound on PKA activity was determined using pep-tag assay. The PKA catalytic subunit was incubated with the candidate compound for $15 \mathrm{~min}$, and then with the PKA substrate. The phosphorylation-induced changes in the electronic charge of the peptide were assessed by electrophoresis after $30 \mathrm{~min}$ of incubation. The intensity of bands was quantified and the relative activity of PKA at each dose was calculated (Figs. 3C, D). We found that the candidate compound decreased the percentage of phosphorylated PKA substrate dose-dependently. The $\mathrm{IC}_{50}$ value of the candidate compound in this assay was $183 \mu \mathrm{M}$, which was calculated by fitting of the Hill equation to the dose-response data. Since the $\mathrm{IC}_{50}$ of kinase is affected by ATP concentration, we performed the same assay using different concentration of ATP $(1,0.2 \mathrm{~mm})$ and found better $\mathrm{IC}_{50}$ $(56 \mu \mathrm{M})$ at low ATP (Fig. 3E). These results suggest that the candidate compound is an ATP-competitive PKA inhibitor.

Then, the inhibitory effect of the candidate compound on the activity of $\mathrm{AC}$, the upstream enzyme of PKA, was determined. HEK293T cells were treated with the candidate compound $(200 \mu \mathrm{M})$ for $1 \mathrm{~h}$; subsequently, forskolin $(10 \mu \mathrm{M})$ was added to the cells and the cells were harvested $15 \mathrm{~min}$ after the treatment. The concentration of cAMP determined by an enzyme-linked immunosorbent assay (ELISA)-based method was markedly increased following forskolin treatment even in the candidate-compound-treated cells (Fig. 3F). This means that the candidate compound is not an $\mathrm{AC}$ inhibitor.

We also examined the effect of the candidate compound $(10 \mu \mathrm{M})$ on cell viability using MTT assay. The MTT signal of HEK293T cells treated with the candidate compound for $24 \mathrm{~h}$ was not significantly different from that of the control, which
A)

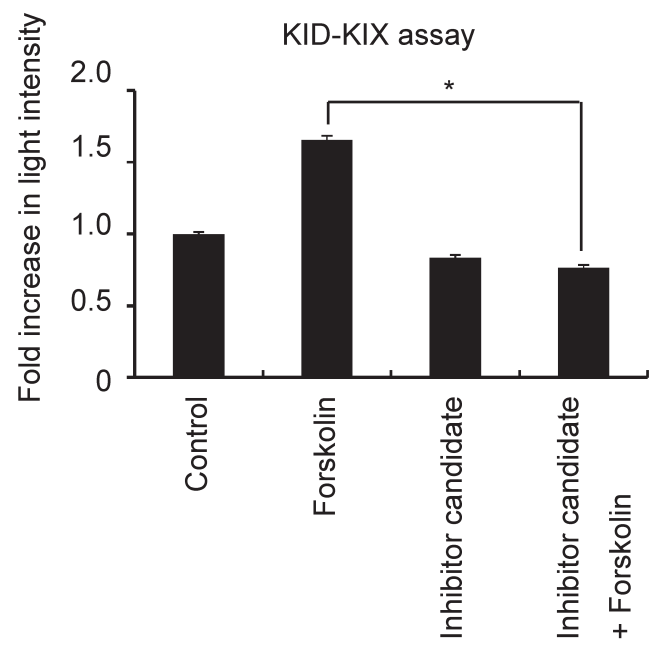

B)

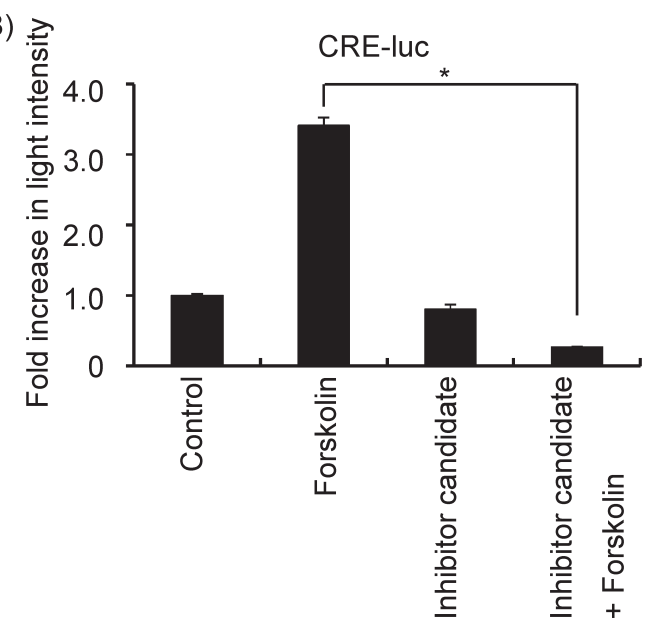

C)

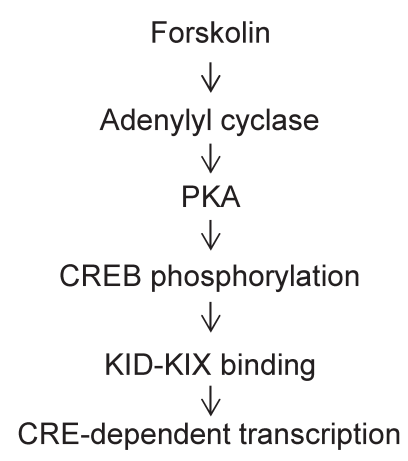

Fig. 2. Reexamination of Inhibitory Effect of Candidate Compound on the Assays Used in the Screening

A) Inhibition of increase in intensity of light emission induced by forskolin was reexamined in cells expressing KID-KIX probe proteins. Data are presented as the mean \pm S.E.M., $n=4$. $* p<0.05$, with Student's $t$-test. B) Suppression of CREdependent gene expression by the candidate compound was verified. Data are presented as the mean \pm S.E.M., $n=4$. ${ }^{*} p<0.05$, with Student's $t$-test. C) Schema of AC/PKA/CREB pathway.

indicated the candidate compound at this concentration did not have toxicity (Fig. 4A). This result means that suppression of the forskolin-induced increase in light emission intensity detected in Figs. 2A and B was not due to cell death caused by the toxicity of the candidate compound. We tested whether the expression levels of c-fos mRNA, which is known to be affected by CREB activity. HEK293T cells were treated with the candidate compound $(10 \mu \mathrm{M})$ for $2 \mathrm{~h}$ and subsequently with 
A)

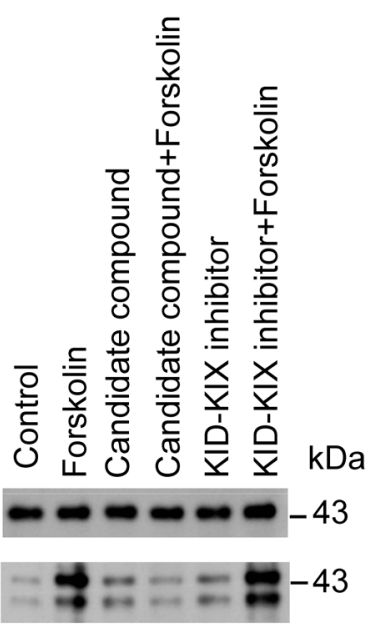

B)

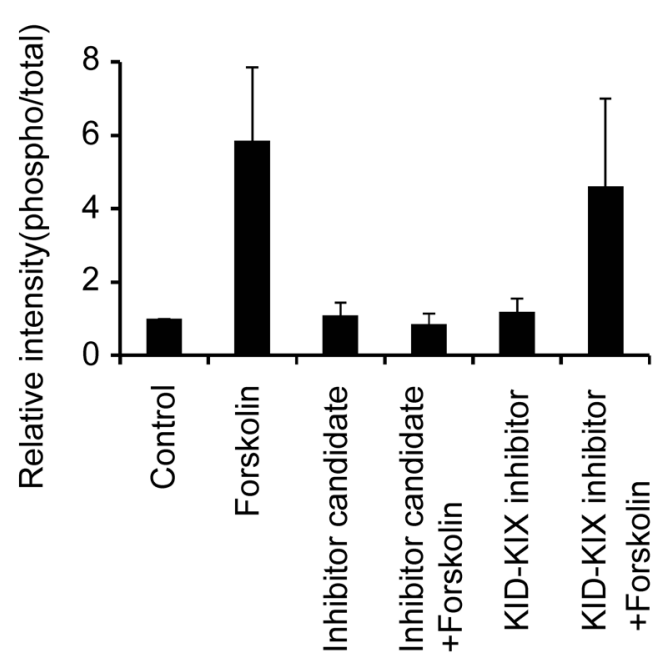

C)

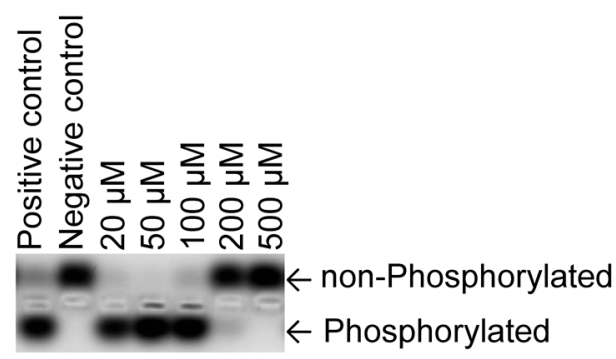

D)

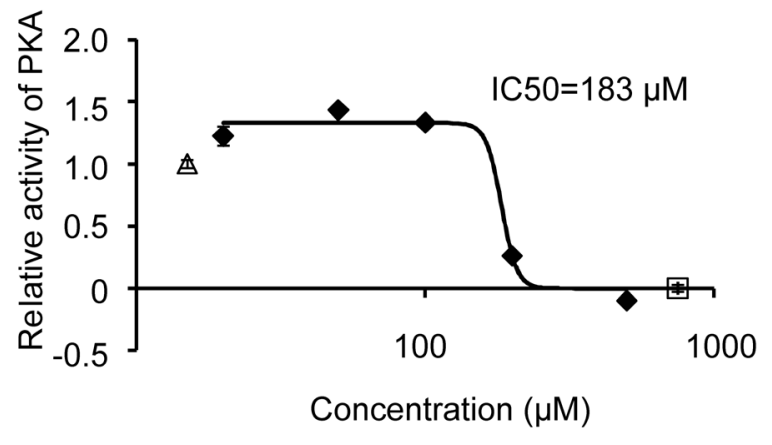

E)

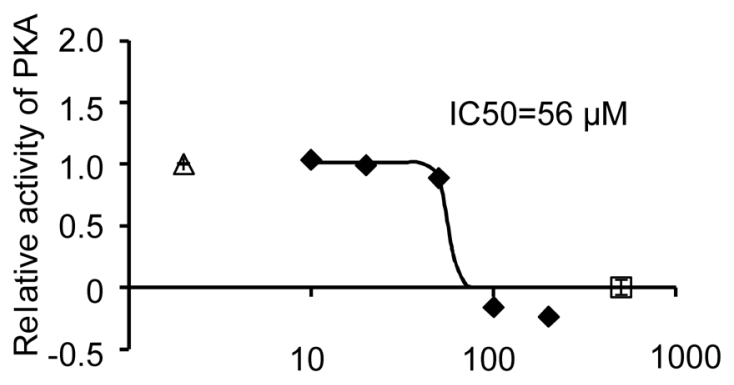

F)

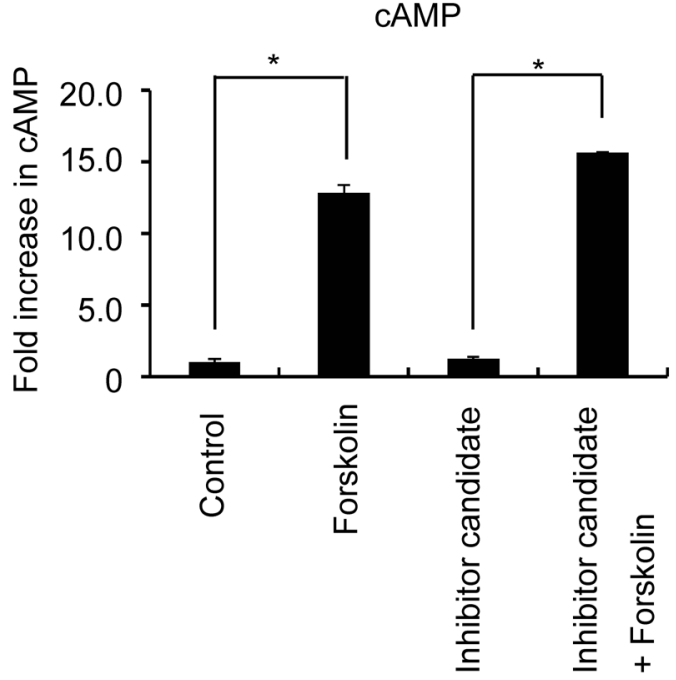

Fig. 3. Inhibitory Effect of Candidate Compound on Enzymes in AC/PKA/CREB Pathway

A) The inhibitory effect of the candidate compound on endogenous CREB phosphorylation. The cells were treated with the candidate compound (10 $\mu \mathrm{M})$ and KID-KIX inhibitor $(10 \mu \mathrm{M})$ for $2 \mathrm{~h}$ and then with forskolin $(10 \mu \mathrm{M})$ for $4 \mathrm{~h}$. And the proteins were analyzed by Western blotting. Upper and lower arrows represent the total and phosphor-CREB band, respectively. The locations of molecular size markers are shown on the right side. B) Summary of Western blotting. The vertical axis represents the phosphorylation level of CREB relative to that of total CREB. Data are the mean \pm S.E.M., $n=4$. C) Image of PKA activity assay. The PKA substrate was incubated with the PKA catalytic subunit in a buffer containing $20 \mathrm{~mm}$ Tris $\mathrm{pH} 7.4,10 \mathrm{~mm} \mathrm{MgCl}_{2}$ and $1 \mathrm{~mm}$ ATP with or without the candidate compound, and phosphorylated and nonphosphorylated substrates were separated by electrophoresis. The upper and lower bands represent the nonphosphorylated and phosphorylated substrate, respectively. The concentrations of the candidate compound are shown on top of the image. D) Summary of inhibitory effect of candidate compound on PKA activity in 1 mm ATP. ( $)$ represents candidate compound, $(\square)$, the negative control without PKA, and $(\triangle)$ the positive control without the candidate compound. Solid line represents fitted curve for the candidate compound. Data are presented as relative activity to the positive control \pm S.E.M., $n=4$. E) $\mathrm{IC}_{50}$ value in the lower concentration $\mathrm{ATP}(0.2 \mathrm{~mm})$. The inhibitory effect of the candidate compound was assessed in a buffer containing $20 \mathrm{~mm}$ Tris pH $7.4,10 \mathrm{~mm} \mathrm{MgCl}_{2}$ and $0.2 \mathrm{~mm} \mathrm{ATP}$. ( $)$ represents candidate compound, ( $\square$ ), the negative control without PKA, and $(\triangle)$ the positive control without the candidate compound. Solid line represents fitted curve for the candidate compound. Data are presented as relative activity to the positive control \pm S.E.M., $n=4$. F) Inhibitory effect of candidate compound on increase in cAMP up-regulation induced by forskolin. The vertical axis represents the cAMP concentration relative to the control. Data are the mean \pm S.E.M., $n=4$. ${ }^{*} p<0.05$, with Student's $t$-test. 
A)

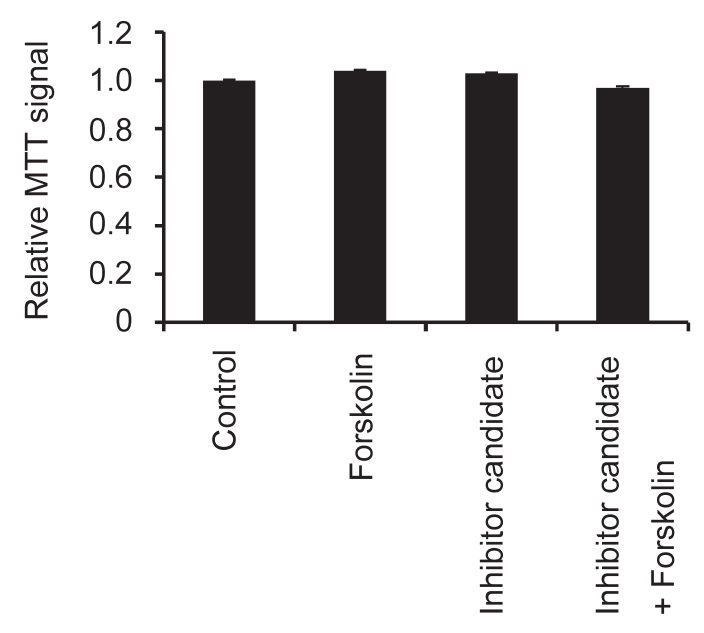

B)

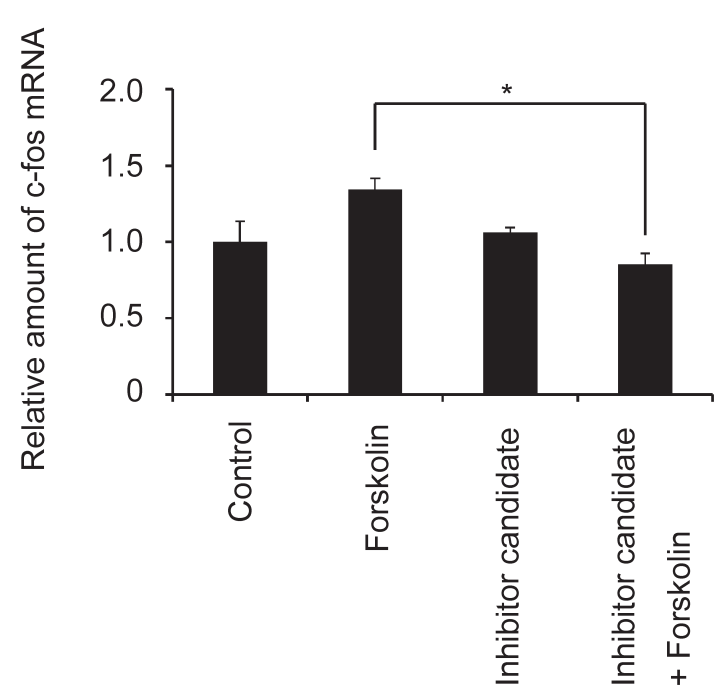

Fig. 4. Effect of Candidate Compound on Cell Viability

A) Viability of HEK293T cells treated with or without the candidate compound was determined by MTT assay. The vertical axis represents viability of treated cells relative to that of the control. Data are presented as relative values to the control \pm S.E.M., $n=4$. B) Comparison of $\mathrm{c}$-fos mRNA expression in candidate compound-treated cells and control cells using quantitative PCR. Data are presented as the mean \pm S.E.M., $n=4$. ${ }^{*} p<0.05$, with Student's $t$-test.

forskolin $(10 \mu \mathrm{M})$ for $1 \mathrm{~h}$. We performed a quantitative PCR using mRNA sample from the candidate compound-treated cells and control, and found the up-regulation of c-fos mRNA induced by forskolin was cancelled by the candidate compound (Fig. 4B). This result supports the inhibitory effect of the candidate compound on CREB phosphorylation pathway.

PKA consists of regulatory and catalytic subunits and its activation is induced by an increase in the concentration of cAMP. Once regulatory subunits are activated by the binding of cAMP, catalytic subunits are released from regulatory subunits and phosphorylate their substrates. There are several types of PKA inhibitor that differ in kinetics and binding sites. H89 and KT5720 are considered to competitively bind to the ATP binding site of the catalytic subunit of PKA. ${ }^{11,13)}$ The protein kinase inhibitor peptide (PKI) binds to the non-ATPbinding site of the catalytic subunit to inhibit the PKA activity. ${ }^{14)}$ Rp-cAMP is an antagonist of the cAMP-binding site of the PKA regulatory subunit and prevents the catalytic subunit from being released. ${ }^{15)}$ The candidate compound identified in the current study may act as an antagonist that binds the ATP binding site of the catalytic subunit, since the candidate compound was a relatively small molecule (MW: 343.44 ) and inhibited the activity of the PKA catalytic subunit ATP concentration dependently directly in vitro (Figs. 3C, D, E). Mutation of the PKA gene that prevents the interaction between the catalytic and regulatory subunits is frequently observed in the patients of corticotropin-independent Cushing's syndrome. As a result, a constitutively active PKA catalytic subunit is generated, which is considered to be a cause of the disease. ${ }^{16)}$ The inhibitors that bind the catalytic subunit directly are expected to be medicines for such a disease.

The $\mathrm{IC}_{50}$ value $(56 \mu \mathrm{M})$ of the candidate compound for PKA activity (Fig. 3E) was higher than the concentration $(10 \mu \mathrm{M})$ used in the first and second screening. The inhibitory effect of the candidate compound may be higher in the cultured living cell used in the KID-KIX screening step. The $\mathrm{IC}_{50}$ of a kinase inhibitor usually varies depending on experimental condition. For example, the $\mathrm{IC}_{50}$ value of KT5720, a known PKA inhibitor, varies from 56 to $3 \mu \mathrm{M}$ depending on experimental condition. ${ }^{17)}$ In the current study, we also found the $\mathrm{IC}_{50}$ was changed ATP-dependently (Figs. 3D, E).

Many PKA inhibitors have side effects such as inhibition of other types of kinases. For example, KT5720 inhibits mitogen-activated protein kinase (MAPK), ${ }^{17}$ and H89 inhibits rho-associated kinase (ROCK). ${ }^{18)}$ These side effects make it difficult to use the inhibitors for medical purposes, and remind us that screening and developing novel PKA inhibitors are still demanded to find specific PKA inhibitors. In the current study, we could not determine whether the candidate compound is specific to PKA, further analysis will be needed to determine the target specificity and kinetics of the candidate compound.

Acknowledgments We thank Drs. Tetsuo Nagano and Takayoshi Okabe for helpful advice. We also thank Ms. Ayumi Yoto for technical assistance. This work was supported by PRESTO of the Japan Science and Technology Corporation (JST, No. 2602), Japan Society for the Promotion of Science (JSPS, Nos. 25350998 and 23650231), and Platform for Drug Discovery, Informatics, and Structural Life Science from the Ministry of Education, Culture, Sports, Science and Technology of Japan.

Conflict of Interest The authors declare no conflict of interest.

\section{REFERENCES}

1) Yin JC, Tully T. CREB and the formation of long-term memory. Curr. Opin. Neurobiol., 6, 264-268 (1996).

2) Carlezon WA Jr, Duman RS, Nestler EJ. The many faces of CREB. Trends Neurosci., 28, 436-445 (2005).

3) Muschamp JW, Van't Veer A, Parsegian A, Gallo MS, Chen M, Neve RL, Meloni EG, Carlezon WA Jr. Activation of CREB in the nucleus accumbens shell produces anhedonia and resistance to extinction of fear in rats. J. Neurosci., 31, 3095-3103 (2011).

4) Briand LA, Blendy JA. Molecular and genetic substrates linking stress and addiction. Brain Res., 1314, 219-234 (2010).

5) James MA, Lu Y, Liu Y, Vikis HG, You M. RGS17, an overex- 
pressed gene in human lung and prostate cancer, induces tumor cell proliferation through the cyclic AMP-PKA-CREB pathway. Cancer Res., 69, 2108-2116 (2009).

6) Siu YT, Jin DY. CREB - a real culprit in oncogenesis. FEBS J., 274, 3224-3232 (2007).

7) Coxon A, Rozenblum E, Park YS, Joshi N, Tsurutani J, Dennis PA, Kirsch IR, Kaye FJ. Mect1-Maml2 fusion oncogene linked to the aberrant activation of cyclic AMP/CREB regulated genes. Cancer Res., 65, 7137-7144 (2005).

8) Mano H, Ishimoto T, Okada T, Toyooka N, Mori H. Discovery of novel adenylyl cyclase inhibitor by cell-based screening. Biol. Pharm. Bull., 37, 1689-1693 (2014).

9) Ozawa T, Kaihara A, Sato M, Tachihara K, Umezawa Y. Split luciferase as an optical probe for detecting protein-protein interactions in mammalian cells based on protein splicing. Anal. Chem., 73, 2516-2521 (2001).

10) Ishimoto $T$, Mano $H$, Ozawa $T$, Mori H. Measuring CREB activation using bioluminescent probes that detect KID-KIX interaction in living cells. Bioconjug. Chem., 23, 923-932 (2012).

11) Murray AJ. Pharmacological PKA inhibition: all may not be what it seems. Sci. Signal., 1, re4 (2008).

12) Best JL, Amezcua CA, Mayr B, Flechner L, Murawsky CM, Emerson B, Zor T, Gardner KH, Montminy M. Identification of smallmolecule antagonists that inhibit an activator: coactivator interac- tion. Proc. Natl. Acad. Sci. U.S.A., 101, 17622-17627 (2004).

13) Lochner A, Moolman JA. The many faces of H89: a review. Cardiovasc. Drug Rev., 24, 261-274 (2006).

14) Dalton GD, Dewey WL. Protein kinase inhibitor peptide (PKI): a family of endogenous neuropeptides that modulate neuronal cAMP-dependent protein kinase function. Neuropeptides, 40, 23-34 (2006).

15) de Wit RJ, Hekstra D, Jastorff B, Stec WJ, Baraniak J, Van Driel R, Van Haastert PJ. Inhibitory action of certain cyclophosphate derivatives of cAMP on cAMP-dependent protein kinases. Eur. $J$. Biochem., 142, 255-260 (1984).

16) Sato $Y$, Maekawa $S$, Ishii R, Sanada M, Morikawa T, Shiraishi $Y$, Yoshida K, Nagata Y, Sato-Otsubo A, Yoshizato T, Suzuki H, Shiozawa Y, Kataoka K, Kon A, Aoki K, Chiba K, Tanaka H, Kume H, Miyano S, Fukayama M, Nureki O, Homma Y, Ogawa S. Recurrent somatic mutations underlie corticotropin-independent Cushing's syndrome. Science, 344, 917-920 (2014).

17) Davies SP, Reddy H, Caivano M, Cohen P. Specificity and mechanism of action of some commonly used protein kinase inhibitors. Biochem. J., 351, 95-105 (2000).

18) Sandvig A, Berry M, Barrett LB, Butt A, Logan A. Myelin-, reactive glia-, and scar-derived CNS axon growth inhibitors: expression, receptor signaling, and correlation with axon regeneration. Glia, $\mathbf{4 6}$, 225-251 (2004). 